Z. Klin. Chem. Klin. Biochem.

12. Jg. 1974 , S. $449-452$

\title{
Galaktokinaseaktivität in Erythrocyten von Patienten mit Down-Syndrom ${ }^{1}$ )
}

\author{
Von Sabine Lippross und F. Menne
}

\begin{abstract}
Aus der Abteilung für Stoffwechselforschung (Vorstand: Prof. Dr. F. Menne) des Physiologisch-Chemischen Institutes der Universität Münster
\end{abstract}

(Eingegangen am 4. Februar/5. August 1974)

Die bisher einzig von Donnell et al. ((1965), Lancet I, 553) mit ciner Isotopenmethode durchgeführten Untersuchungen über die Galaktokinaseaktivität (EC 2.7.1.6) im Erythrocytenhämoly sat von Mongoloiden wurden mittels einer enzymatischen Bestimmungstechnik überprüft. Die von Donnell et al. ermittelte 20\%ige Aktivitätserhöhung bei mongoloiden Kindern gegenüber der Norm wurde als signifikant bestätigt, obwohl sich die Einzelwerte bei mongoloiden und normalen Probanden beträchtlich überschnitten. Alter und Geschlecht hatten nachweislich keinen Einfluß auf die Untersuchungsergebnisse. Als ätiologische Faktoren der erhöhten Enzymaktivität wurden sowohl der sogenannte „Gene Dose Effect" als auch eine allgemeine oder auf die roten Blutzellen beschränkte Reifungshemmung diskutiert und abgelehnt.

\section{Quantitative enzymic determination of galactokinase activity in the erythrocyte haemolysate of patients with Down's Syndrome}

The galactokinase activity (EC 2.7.1.6) in the erythrocyte haemolysate of mongoloids has been the subject of one investigation with the aid of an isotopic method, by Donnell et al. ((1965), Lancet $I, 553)$. This has now been checked with the aid of an enzymic determination, modified according to Gesche \& Menne. The $20 \%$ increase above the norm of the activity in mongoloid children reported by Donnell et al. was confirmed as significant, although the individual values for mongoloids and normal probands showed considerable overlap. Age and sex had no detectable influence on the results. The so-called "gene dose effect" and an inhibition of maturation, of a general nature or limited to the erythrocytes, are discussed and rejected as possible aetiological factors in the increased enzyme activity.

Je nach angewandter Technik voneinander abweichende Ergebnisse bei Enzymbestimmungen im Blut von Patienten mit Down-Syndrom warfen die Frage auf, ob die von Donnel et al. (1) gefundene Erhöhung der Galaktokinaseaktivität im Erythrocytenhämolysat sich bestätigen ließe. Ähnliche Untersuchungen über die UDPG-Transferase (EC 2.7.7.12) (2) gaben Anlaß zu vermuten, daß die Isotopenmethode obiger Autoren zu niedrige Werte erzielte, verglichen mit der enzymatischen Bestimmung (3), die auf Grund ihrer Direktheit und einfachen Handhabung ein Höchstmaß an Genauigkeit bietet.

\section{Methodik}

\section{Probanden}

Untersucht wurden dreißig 6-14jährige Kinder, bei denen klinisch eindeutig ein Down-Syndrom festzustellen war. Zusätzlich wurde bei sieben der Probanden die Diagnose cytologisch erhärtet. Die Altersspanne 6-14 Jahre wurde gewählt im Hinblick auf folgende zwei Gesichtspunkte:

Im Alter von 3 bis 6 Jahren stellt sich die Galaktokinaseaktivität von Kindern auf ein Niveau ein, das während des weiteren Lebens annähernd beibehalten wird. Erst vom sechsten Lebensjahr an ist also eine Wertkonstanz gewährleistet, die den Vergleich innerhalb einer größeren Gruppe erlaubt.

Die obere Altersgrenze von vierzehn Jahren wurde gewählt, um möglichst weitgehende Homogenität bezüglich der auf die $\mathrm{Pa}$ - tienten einwirkenden Umweltfaktoren zu erzielen. Die Probanden waren sämtlich Schüler der Max-Wittmann-Schule Dortmund, einer Tagesstätte für geistig behinderte Kinder.

Als Kontrollgruppe dienten dreißig gesunde gleichaltrige Probanden. Für die Untersuchung wurde jedem mongoloiden ein normales Kind gleichen Alters und Geschlechts zugeordnet. Weder die Mongoloiden noch die normalen Vergleichspersonen wiesen zur Zeit der Untersuchung irgendeinen Anhaltspunki für Infekte oder andere unser Problem möglicherweise beeinflussende Krankheiten auf. Die normalen Probanden waren $\mathrm{Pa}$ tienten der kinderchirurgischen Abteilung der Städtischen Krankenanstalten in Dortmund, der HNO- sowie der Chirurgischen Universitätsklinik in Münster.

Um den von mehreren Autoren bei Lagerung des Blutes beobachteten Aktivitätsverlust des Enzyms $(3,4)$ auszuschließen, wurde das Blut jeweils am Tag der Entnahme sofort aufgearbeitet.

\section{Prinzip}

Das Maß für die Aktivität des Enzyms Galaktokinase

(EC 2.7.1.6) ist die in der Zeiteinheit verbrauchte Menge Galaktose.

Nach Abstoppen der Meß-Reaktion wird die nicht-phosphorylierte Galaktose mit Hilfe der Galaktose-Dehydrogenase (EC 1.1.1.48) im optischen Test bestimmt.

$$
\mathrm{Gal}+\mathrm{ATP} \stackrel{\text { Galaktokinase }}{\longrightarrow} \mathrm{Gal}-1-\mathrm{P}+\mathrm{ADP}
$$

1) Durchgeführt mit freundlicher Unterstützung des Ministerpräsidenten des Landes Nordrhein-Westfalen - Landesamt fuir Forschung. 
Die nicht-phosphorylierte Galaktose wird umgesetzt zu

$\begin{aligned} \mathrm{Gal} & +\mathrm{NAD}+\underset{+}{\stackrel{\text { Galaktose-Dehydrogenase }}{\longrightarrow} \text { Galaktonolacton }+} \\ & +\mathrm{NADH}+\mathrm{Hg}^{++}\end{aligned}$

Die methodischen Einzelheiten werden nach den von Gesche und Menne (3) gegebenen Richtlinien durchgeführt, wobei sich Doppelbestimmungen der Leerwerte als unnötig erwiesen.

Berechnung

Die umgesetzte Galaktosemenge ist der gebildeten NADH-Menge äquivalent und läßt sich durch folgende Rechnung ermitteln:

$\Delta \mathrm{E} \times \mathrm{k} \times \mathrm{c}=\mu \mathrm{mol} \mathrm{Gal}-1-\mathrm{P} / \mathrm{h} \times \mathrm{ml}$ Erythrocyten

Die von Donnell et al. angegebene Maßeinheit ( $\mu \mathrm{mol} \mathrm{Gal} \mathrm{-} 1$ $-\mathrm{P} / \mathrm{h} \times \mathrm{ml}$ Erythrocyten) wurde auch in der vorliegenden Arbeit verwandt, um einen Vergleich der absoluten Werte zu gestatten. Die heute gebräuchliche Angabe in Enzymeinheiten bezogen auf $\mathrm{g} \mathrm{Hb}(\mu \mathrm{mol} \mathrm{Gal} \mathrm{-} 1-\mathrm{P} / \mathrm{min} . \times \mathrm{g} \mathrm{Hb})$ ergäbe sich durch multiplizieren der Ergebnisse mit dem Faktor

$\frac{1}{60 \times 34}=\frac{1}{2040}$

$\Delta E=\left(E_{2}-E_{1}-E_{0}\right)_{L}-\left(E_{2}-E_{1}-E_{0}\right)_{R}$

$\mathrm{L}=$ Leerwert

$\mathrm{R}=$ Reaktionswert

$\mathrm{E}_{0}=$ Eigenextinktion der Galaktose-Dehydrogenase

$E_{1}=$ Anfangsextinktion von Reaktions- und Leer-Ansatz

$E_{2}=$ Extinktion nach Zugabe von Galaktose-Dehydrogenase

Die Konstante k ergibt sich aus dem Bestimmungsansatz und setzt sich zusammen aus

$\mathrm{k}=\frac{\mathrm{V} \times \mathrm{F}}{\epsilon \times \mathrm{d} \times \mathrm{v} \times \mathrm{T}}=10,5$

$\mathrm{V}=$ Testvolumen der Küvette $(3,32 \mathrm{ml})$

$\mathrm{F}=$ Verdünnungsfaktor für Hämolysat $(2,1)$

$\epsilon \quad=$ mikromolarer Extinktionskoeffizient von NAD

$\left(3,3 \mathrm{~cm}^{2} / \mu \mathrm{mol}\right.$ bei $\left.366 \mathrm{~nm}\right)$

$\mathrm{d}=$ Schichtdicke in $\mathrm{cm}(1 \mathrm{~cm})$

$\mathrm{v}=$ Filtratvolumen $(0,2 \mathrm{ml})$

$T=$ Inkubationszeit $(1 \mathrm{~h})$

Unter der Annahme von Normochromität und nicht von der Norm abweichendem Einzelvolumen der Erythrocyten enthält 11 Erythrocyten $340 \mathrm{~g} \mathrm{Hb}$.

Daraus ergibt sich $c$ als

$c=\frac{34}{\mathrm{Hb}_{\mathrm{z} \text { Hämolysat }}}$

Der Hämoglobingehalt wurde bestimmt als Hämiglobincyanid nach Drabkin.

\section{Ergebnisse}

\section{Daten}

Die beschriebenen Untersuchungen brachten Ergebnisse, die in der folgenden Tabelle zusammengefaßt sind:
Tab. 1. Enzymaktivitäten bei Patienten mit Down-Syndrom und normalen Probanden.

Aktivität [ $\mu \mathrm{mol} \mathrm{Gal} \mathrm{-} \mathrm{1} \mathrm{-} \mathrm{P/h} \times$ ml Erythrocyten], Alter [Jahre]

\begin{tabular}{lllll}
\hline Alter & $\begin{array}{l}\text { Down- } \\
\text { Syndrom }\end{array}$ & Normale & $\begin{array}{l}\text { Down- } \\
\text { Syndrom }\end{array}$ & Normale \\
\hline 6 & 0,513 & 0,550 & 0,768 & 0,438 \\
& & & 0,658 & 0,485 \\
7 & 0,507 & 0,580 & 0,644 & 0,570 \\
8 & 0,604 & 0,526 & & \\
9 & 0,759 & 0,499 & & \\
& 0,685 & 0,463 & 0,559 & 0,635 \\
& & & 0,636 & 0,561 \\
10 & & & 0,781 & 0,485 \\
11 & 0,647 & 0,504 & 0,715 & 0,679 \\
& 0,775 & 0,716 & 0,575 & 0,573 \\
12 & 0,578 & 0,623 & 0,512 & 0,485 \\
& 0,485 & 0,481 & 0,670 & 0,476 \\
& & & 0,689 & 0,454 \\
13 & 0,776 & 0,605 & 0,685 & 0,558 \\
& 0,556 & 0,561 & 0,591 & 0,535 \\
& & & 0,647 & 0,453 \\
14 & 0,637 & 0,716 & 0,513 & 0,510 \\
& 0,667 & 0,445 & 0,676 & 0,505 \\
\hline
\end{tabular}

Der für die Gruppe der Mongoloiden errechnete Mittelwert beträgt

$\overline{\mathrm{X}}_{\mathrm{M}}=0,640 \mu \mathrm{mol} \mathrm{Gal}-1-\mathrm{P} / \mathrm{h} \times \mathrm{ml}$ Erythrocyten

mit einer Standardabweichung

$s_{M}= \pm 0,087 \mu \mathrm{mol} \mathrm{Gal}-1-\mathrm{P} / \mathrm{h} \times$ ml Erythrocyten

Der für die normale Vergleichsgruppe errechnete Mittelwert beträgt

$\overline{\mathrm{x}}_{\mathrm{N}}=0,538 \mu \mathrm{mol} \mathrm{Gal}-1-\mathrm{P} / \mathrm{h} \times \mathrm{ml}$ Erythrocyten

mit einer Standardabweichung

$\mathrm{s}_{\mathrm{N}}= \pm 0,077 \mu \mathrm{mol} \mathrm{Gal}-1-\mathrm{P} / \mathrm{h} \times \mathrm{ml}$ Erythrocyten

Der mittlere Wert der Galaktokinaseaktivität bei den Patienten mit Down-Syndrom liegt somit um 20\% höher als bei den normalen gleichaltrigen Kindern.

\section{Statistik}

Die Untersuchungsergebnisse wurden einem $t$-Test unterzogen, der die 20\%ige Erhöhung des Mittelwertes $\bar{x}_{M}$ gegenüber $\overline{\mathbf{x}}_{\mathrm{N}}$ als signifikant darstellte. Da die ermittelten Werte nicht mit Sicherheit als normalverteilt anzusehen sind (Asymmetrie der Zahlenverteilung in Abb. 3), wurde zusätzlich ein von Verteilungen unabhängiger Man/Whitney-Test angewandt, der den t-Test in seiner Aussage bestätigte.

Der Einfluß des Geschlechtes auf die Galaktokinaseaktivität wurde durch eine Varianzanalyse überprüft und verneint. 


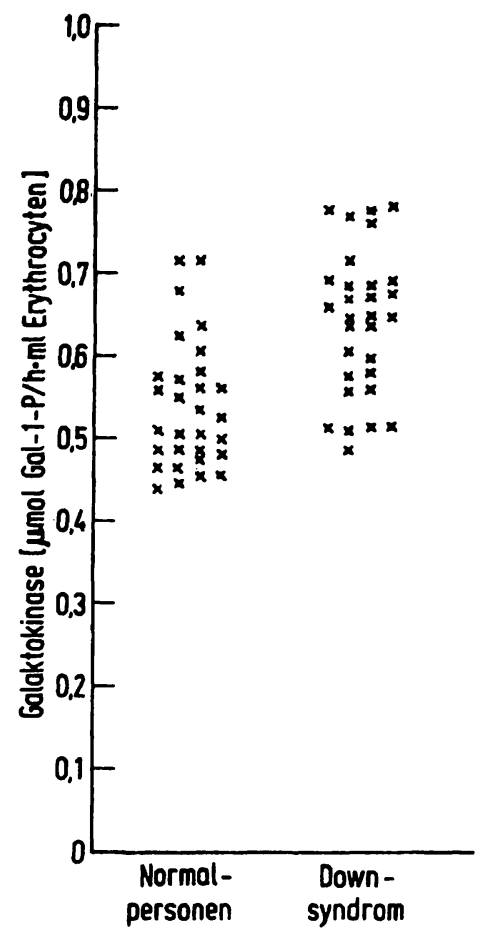

Abb. 1. Datenverteilungsskizze der Galaktokinaseaktivität in Erythrocyten von Normalpersonen und Patienten mit Down-Syndrom.

Die Streuung der Werte innerhalb der Gruppen gleichen Alters zeigte, daß zwischen dem Alter der Probanden und den Untersuchungsergebnissen kein Zusammenhang besteht.

Anhand eines weiteren $t$-Testes wurde nachgewiesen, daß sich die Daten der auf Grund ihres Phänotyps und der klinischen Anżeichen diagnostizierten 23 mongoloiden nicht signifikant von denen der 7 cytogenetisch bestimmten mongoloiden Kindern unterschieden und somit alle als repräsentativ für die Patienten mit DownSyndrom schlechthin gelten dürfen. Die Aussagekraft der Untersuchungsergebnisse im Hinblick auf Reproduzierbarkeit wurde geprüft und bei einem durchschnittlichen Fehler von $0,75 \%$ als genügend hoch befunden.

\section{Diskussion}

Die Galaktokinaseaktivität bei Patienten mit DownSyndrom wurde bisher nur von zwei Arbeitsgruppen untersucht, und zwar von Krone, Wolf, Goedde \& Baitsch (5) und von Donnell, Won, Bergren, Melnyk \& Koch (1), beiden im Jahre 1965. Sinnvoll heranzuziehen für einen Vergleich mit den in der vorliegenden Arbeit ermittelten Werten ist jedoch nur die Arbeit von Donnell et al., die gleichfalls die Erythrocytenenzymaktivität behandelt, während Krone et al. Vollblut zum Gegenstand ihrer Untersuchung machten. Die anfängliche Vermutung, daß die enzymatische Untersuchungsmethode der Galaktokinaseaktivität zu anderen, möglicherweise höheren Ergebnissen führen würde als die von Donnell et al. verwandte Isotopenmethode, erwies sich als nicht richtig. Die Befunde obiger Autoren wurden nach unseren Ermittlungen voll bestätigt. Wohl unterscheiden sich die ermittelten Aktivitäten in ihrer absoluten Größe, nicht jedoch in Relation gesetzt zu den zum Vergleich herangezogenen Normalwerten:

Tab. 2. Vergleich der Galaktokinaseaktivitäten. [ $\mu \mathrm{mol} \mathrm{Gal}-1-\mathrm{P} / \mathrm{h} \times \mathrm{ml}$ Erythrocyten]

\begin{tabular}{llll}
\hline & $\overline{\mathbf{x}}_{\text {Down-Syndrom }}$ & $\overline{\mathbf{x}}_{\text {Normale }}$ & $\begin{array}{l}\overline{\mathrm{x}}_{\text {Down-Syndrom }} \\
\overline{\mathbf{x}}_{\text {Normale }}\end{array}$ \\
\hline $\begin{array}{l}\text { Donnell } \\
\text { et al. }\end{array}$ & 0,360 & 0,300 & $1,2: 1$ \\
$\begin{array}{l}\text { eig. } \\
\text { Unters. }\end{array}$ & 0,640 & 0,538 & $1,2: 1$ \\
\hline
\end{tabular}

Abgesehen von dieser in beiden Arbeiten eindeutig festgestellten Mittelwertserhöhung der Galaktokinaseaktivität um $20 \%$ über die Norm, ergab sich ebenfalls übereinstimmend eine weite Uberschneidung der niedrigsten Werte beim Down-Syndrom mit den normalen. Eine diagnostische Möglichkeit im Sinne einer eindeutigen Abgrenzung mongoloid/normal ist daher im Einzelfall nicht gegeben.

Ein weiterer methodischer Unterschied zwischen den beiden verglichenen Arbeiten besteht bezüglich der Auswahl der Probanden. Obwohl Donnell et al. sich der Variabilität der Galaktokinaseaktivität bis zum Alter von $6 \mathrm{Jahren}$ bewußt waren, untersuchten sie 34 Kinder im Alter von 1-9 Jahren mit einem mittleren Alter von 5,2 Jahren. Die Altersspanne der von uns untersuchten Probanden erstreckte sich aus schon oben angeführten Gründen von 6-14 Jahren und ergab ein mittleres Alter von 10,8 Jahren, so daß die hier ermittelten Ergebnisse bezüglich der Wertkonstanz als gesichert gelten dürfen.

Die Deutung der Ergebnisse als sogenannter „Gene Dose Effect", wie sie vielen Autoren als Arbeitshypothese gedient hat $(5-16)$ muß auch hier, wie bei den meisten der zitierten Autoren, als ursächlicher Faktor für die Aktivitätserhöhung abgelehnt werden. Zum einen ließ sich nicht eine 50\%ige - theoretisch zu erwartende - sondern nur eine 20\%ige Erhöhung nachweisen. Zum anderen ist die Galaktokinase nur eines von verschiedenen Enzymen, die beim Down-Syndrom vermehrt sind, und es ist unwahrscheinlich, daß die Loci für diese Enzyme alle auf dem Chromosom 21 angeordnet sind.

Die Tatsache, daß die frühkindliche Galaktokinaseaktivität deutlich über der bei älteren Kindern beobachteten liegt, könnte zu der Hypothese verleiten, daß die Erhöhung auf eine entweder nur die Erythrocyten betreffende oder aber eine allgemeine körperliche Reifungs- 
störung der Mongoloiden zurückzuführen ist. Auch diese Hypothese muß jedoch sofort verneint werden:

Die Erythrocyten mongoloider Patienten sind morphologisch als regelrecht befunden worden (14). Eine infolge verzögerter allgemeiner Reife erst später eintretende $\mathrm{Ab}$ nahme der Aktıvität hätte sich in einem Wertgefälle von den jüngsten zu den ältesten Probanden ausdrücken müssen. In unseren Untersuchungen zeigte sich die Aktivität jedochals absolut altersunabhängig.

Gegen eine allgemeine verzögerte körperliche Reife spricht auch die äußere Erscheinung der Patienten, die eher den Eindruck einer Frühalterung vermittelt.

\section{Literatur}

1. Donnell, G. N., Won, G., Bergren, W. R., Melnyk, J. \& Koch, R. (1965), Lancet $I, 553$.

2. Nothjungc, J., Most, J., Hass, J. \& Menne, F. (1974), dicsc Z. 12, 59-61.

3. Gesche, M. \& Mcnne, F. (1972), diese Z. 10, 430-433.

4. Ng, W. G., Donncll, G. N. \& Bergren, W. R. (1965), J. Lab. Clin. Med. 66, 115-121.

5. Krone, W., Wolf, U., Goeddc, H. W. \& Baitsch, H. (1965), Ilum. Genct. I, 279-288.

6. Brandt, N. J. (1962), Lancet $I I, 837$.

7. Baikic, A. G. (1962), Lancet II, 937.

8. Lawler, S. D. (1962), Lancet II, 837.

9. Mellman, W. J., Oski, F. A., Tedesco, T. A. \& Harris, H. (1964), Lancet II, 674-675.

10. Stalder, G. R., Bühler, E. M., Bühler, U. K., Egli, F., Richterich, R. \& Colombo, J. P. (1965), Lancet $I, 768$.
11. Baikic, A. G., Loder, P. B., dc Gruchy, G. C. \& Pitt, D. B. (1965), Lancet $I, 412-414$.

12. Reismann, L. E., Kasahara, S., Chung, C. Y., Darnell, A. \& Hall, B. (1966), Lancet $I$, 394-395.

13. Schupisser, R., Joss, E. \& Richterich, R. (1967), Schweiz. Med. Wochenschr. 97, 1540-1542.

14. Pantclakis, S. N., Karaklis, A. G., Alexiou, D., Vardas, E. \& Valaes, T. (1970), Amer. J. Hum. Genct. 22, 184-191.

15. Hsia, D. Y-Y., Justice, P., Smith, G. F., Maywood, Ill., Dowben, R. M. \& Providence, R. I. (1971), Amer. J. Dis. Child. 121, 153-161.

16. Schwarzmeier, J. D., Rett, A., Moser, K. \& Andrlc, M. . (1973), Wien. Klin. Wochenschr. 3, 33-35.
Prof. Dr. F. Menne Physiol. Chem. Inst. 44 Münster Waldeycrstraße 15 